\title{
Hepatic Reactive Lymphoid Hyperplasia and Primary Hepatic Lymphoma: Ultrasound Features and Differentiation Diagnosis
}

\author{
Xiaohui Qiao, MD ${ }^{a, b}$, Kun Chen, MD ${ }^{b}$, Gang Chen, MD, PhD ${ }^{c}$, Liyun Xue, MD ${ }^{a}$, Guangwen Cheng, MD ${ }^{b}$, Hong \\ Ding, MD, PhD ${ }^{a, b, *}$ \\ ${ }^{a}$ Department of Ultrasound, Zhongshan Hospital, Fudan University, Shanghai, China; ${ }^{b}$ Department of Ultrasound, Huashan Hospital, \\ Fudan University, Shanghai, China; ${ }^{c}$ Department of Pathology, Zhongshan Hospital, Fudan University, Shanghai, China \\ Received November 25, 2020; revision received December 21, 2020; accepted December 22, 2020
}

Objective: To investigate the ultrasound features of hepatic reactive lymphoid hyperplasia (HRLH) and primary hepatic lymphoma (PHL), and determine the value of ultrasound on the diagnosis and differentiation of them.
Methods: The ultrasound findings of 11 HRLH lesions and 14 PHL lesions from April 2011 to September 2020 were retrospectively analyzed. The conventional ultrasound and contrast-enhanced ultrasound (CEUS) manifestations of them were compared.

Results: HRLH showed significant female preference than PHL $(100 \%$ and $64.3 \%)(P=0.046)$. There were no significant differences of other demographics between HRLH and PHL patients. On conventional ultrasound, the diameter of HRLH $(16.5 \pm 3.8 \mathrm{~mm})$ was significantly smaller than that of PHL $(48.3 \pm 36.2 \mathrm{~mm})(P=0.008)$, and a regular shape was seen more frequently in HRLH $(90.9 \%)$ than that in PHL $(50 \%)(P=0.038)$. There were no significant differences of other indexes between HRLH and PHL. On CEUS, both HRLH and PHL presented arterial enhancement and washout in early portal phase. But rim enhancement at the beginning of washout was seen more frequently in HRLH $(90.0 \%)$ than that in PHL $(33.3 \%)(P=0.036)$, and HRLH usually displayed hyperenhancement (100\%) in arterial phase while PHL could be hypoenhancement (16.7\%), isoenhancement (33.3\%) and hyperenhancement (50\%) $(P=0.036)$.

Conclusion: Ultrasound can supply valuable imaging evidences for the diagnosis of HRLH and PHL. The ultrasound features including size, shape, degree of arterial phase enhancement and rim enhancement at the beginning of washout can be used for the differentiation of HRLH and PHL.

Key words: Hepatic reactive lymphoid hyperplasia; Primary hepatic lymphoma; Ultrasound

Advanced Ultrasound in Diagnosis and Therapy 2021; 02: 063-072

DOI: $10.37015 / A U D T .2021 .200069$

$\mathrm{H}$ epatic reactive lymphoid hyperplasia (HRLH), also referred to hepatic pseudolymphoma, is an extremely rare benign lesion of the liver [1]. It mainly consists of approximately the same quantity of polyclonal $\mathrm{T}$ and $\mathrm{B}$ lymphocytes with the formation of lymphoid follicles and active germinal centers [2, $3]$. Due to the lack of reports with a large number of cases and insufficient recognition, it is intractable to distinguish HRLH from other focal liver lesions, one of which is primary hepatic lymphoma (PHL).

PHL is a kind of extranodal malignant lymphoma, only localized in the liver without involvement of distant lymph nodes, spleen, bone marrow, other lymphoid tissues and organs. It is characterized by monoclonal lymphocyte proliferation, cell atypia and mitosis [46]. PHL is also an infrequent focal lesion of the liver,

\footnotetext{
* Corresponding author: Department of Ultrasound, Zhongshan Hospital and Huashan Hospital, Fudan University, Shanghai, China. e-mail:ding_hong@fudan.edu.cn unrestricted use, distribution and reproduction in any medium provided that the original work is properly attributed.
} 
accounting for only $0.1 \%$ of liver malignant tumors [7]. For the very different treatment and prognosis of HRLH and PHL, the differentiation diagnosis of them is particularly important.

The differentiation of HRLH and PHL now depends on postoperative pathology, and the preoperative diagnosis is quite difficult. If more comprehensive references can be provided, unnecessary invasive operations will be avoided to a large extent.

Ultrasound is the most popular modality for the screening and diagnosis of focal liver lesions, however, as far as we know, the comparison of ultrasound features between HRLH and PHL has not been reported in detail as yet. Instead, the current studies about them mostly accentuate computed tomography (CT) and magnetic resonance imaging (MRI) manifestations which still cannot provide enough diagnostic information [8-11]. This study is aimed at summarizing the ultrasound features of HRLH and PHL, and providing more valuable imaging evidences for the clinical diagnosis and therapy.

\section{Materials and Methods}

\section{Patients}

This study was approved by the ethics committee of our institute. The data of 11 patients with HRLH and 14 patients with PHL diagnosed from April 2011 to September 2020 were collected. The inclusion criteria: (i) The lesions were all confirmed by liver biopsy or postoperative pathology. (ii) All patients underwent conventional ultrasound or contrast-enhanced ultrasound (CEUS) examination before surgery or biopsy. (iii) The type of PHL was single or multiple type, the diffuse type was not selected, and there was no infiltration of spleen, other organs, distant lymph nodes and peripheral blood. The laboratory tests including alpha fetoprotein (AFP), carcinoembryonic antigen (CEA), carbohydrate antigen 19-9 (CA19-9) and lactate dehydrogenase (LDH) were also carried out for all the patients.

\section{Ultrasound examination}

The ultrasound examination was carried out using the ultrasound device PHILIPS IU 22 and GE LOGIQ E9, the 1-5 MHz convex transducer was selected. The liver was given a systematic scanning under conventional ultrasound, then the lesion was specifically observed. When there were multiple similar lesions, the largest one was selected for imaging. After gray-scale ultrasound, the color Doppler ultrasound was used for blood flow evaluation of the lesion and the resistance index of arterial waveform was measured. Next, the optimal plane was fixed for CEUS examination. The contrast agent used was SonoVue (Bracco, Italy), after dissolving the powder in $5 \mathrm{~mL}$ of saline and fully shaking, $2.4 \mathrm{~mL}$ of contrast agent was transported into the patient via intravenous bolus injection followed by $5 \mathrm{~mL}$ of saline. The change of echogenicity of the lesion and peripheral liver tissue was observed for 4-6 minutes. During the whole examination, the static images and cinematic loops were stored in real time for later analysis and measurement.

\section{Image analysis}

The image analysis was performed by two radiologists (with 5-10 years of experiences in ultrasound imaging of the liver) without knowing the pathological results, the difference was resolved in consensus. For conventional ultrasound, the qualitative indexes included the lesion's location (right / left / caudate lobe), whether subcapsular lesion or not, echotexture (anechoic / hypoechoic / isoechoic / hyperechoic), the presence of posterior echo enhancement, shape (regular / irregular), boundary (clear / not clear), and color Doppler flow signal (no / dotted / linear / rich). Rich color Doppler flow signal meant more than three linear blood flow signals. The quantitative indexes included the maximum diameter and resistance index. For CEUS, the qualitative indexes included the degree of arterial phase enhancement (hypoenhancement / isoenhancement / hyperenhancement) and the presence of rim enhancement at the beginning of washout. The quantitative indexes included washin time, time to peak and washout time of the lesion. The vascular phases and timing were consistent with American College of Radiology Contrast-Enhanced Ultrasound Liver Imaging Reporting and Data System: arterial phase occurred from $10-20 \mathrm{~s}$ to $30-45 \mathrm{~s}$ after contrast injection, portal venous phase lasted from $30-45 \mathrm{~s}$ to $120 \mathrm{~s}$ after contrast injection and late phase lasted from $120 \mathrm{~s}$ to microbubble disappearance at about 4-6 minutes. Hypoenhancement, isoenhancement and hyperenhancement were defined as decreased, the same and increased enhancement degree compared with the surrounding liver tissue. Washin time, time to peak and washout time were determined as the time from contrast injection to the beginning of lesion enhancement, the peak of lesion enhancement and the reduction of lesion enhancement [12].

\section{Pathological examination}

All 11 HRLH lesions were confirmed by postoperative pathology, 3 PHL lesions were confirmed by liver biopsy, and the other 11 PHL lesions were confirmed by postoperative pathology. The hematoxylin eosin and immunohistochemistry staining were applied in all the cases. Besides, some patients also underwent Epstein-Barr virus-encoded small RNA (EBER) analysis, reticulum fiber staining, gene rearrangement and dual- 
color fluorescence in situ hybridization (DC-FISH) analysis to obtain accurate pathological results.

\section{Statistical Analysis}

The SPSS 22.0 software (SPSS Inc, Chicago, USA) was applied for statistical analysis. The measurement data were expressed as mean value \pm standard deviation $(\bar{x} \pm s)$. The differences of quantitative variables were compared with the Student $t$ test when normality and homogeneity of variance assumptions were satisfied, otherwise, the Wilcoxon rank sum test was used. The differences of qualitative variables were compared with $\chi^{2}$ test or Fisher's exact test. $P<0.05$ was considered to indicate significance.

\section{Results}

\section{General information}

The demographics of the patients were shown in
Table 1. Compared with PHL, HRLH showed significant female preference $(100 \%$ and $64.3 \%)(p=0.046)$. The mean age of patients with HRLH was not significantly different from that of patients with PHL $(p=0.100)$. The HRLH lesions were accidentally detected by health checkups or during the examination for other diseases, and none of the patients with HRLH had special medical history. Among the 14 patients with PHL, 11 (78.6\%) cases were detected incidentally, $2(14.3 \%)$ cases were found for abdominal discomfort and $1(7.1 \%)$ case for anorexia and fatigue. As for the medical history, 9 $(64.3 \%)$ patients had no special liver related diseases, $1(7.1 \%)$ patient had schistosomiasis, $1(7.1 \%)$ patient had hepatitis A virus infection and 3 (21.4\%) patients had hepatitis B virus infection. There were no significant differences of serum AFP, CEA, CA19-9 and LDH between HRLH and PHL patients $(P>0.05)$, and the average levels were all within the normal range.

Table 1 Baseline characteristics of HRLH and PHL patients

\begin{tabular}{|c|c|c|c|}
\hline Item & $\operatorname{HRLH}(n=11)$ & $\operatorname{PHL}(n=14)$ & $P$ value \\
\hline \multicolumn{4}{|l|}{$\operatorname{Sex}(n, \%)$} \\
\hline Female & $11(100.0)$ & $9(64.3)$ & 0.046 \\
\hline Male & $0(0.0)$ & $5(35.7)$ & \\
\hline Age (y) & $49 \pm 11$ & $58 \pm 13$ & 0.100 \\
\hline \multicolumn{4}{|l|}{ Complaint $(n, \%)$} \\
\hline No & $11(100.0)$ & $11(78.6)$ & 0.487 \\
\hline Abdominal discomfort & $0(0.0)$ & $2(14.3)$ & \\
\hline Anorexia and fatigue & $0(0.0)$ & $1(7.1)$ & \\
\hline \multicolumn{4}{|l|}{ Medical history $(n, \%)$} \\
\hline No & $11(100.0)$ & $9(64.3)$ & 0.147 \\
\hline Schistosomiasis & $0(0.0)$ & $1(7.1)$ & \\
\hline Hepatitis A virus infection & $0(0.0)$ & $1(7.1)$ & \\
\hline Hepatitis B virus infection & $0(0.0)$ & $3(21.4)$ & \\
\hline $\operatorname{AFP}(<20 \mathrm{ng} / \mathrm{mL})$ & $2.7 \pm 1.5$ & $3.1 \pm 2.5$ & 0.794 \\
\hline $\mathrm{CEA}(<5 \mathrm{ng} / \mathrm{mL})$ & $1.2 \pm 1.0$ & $1.4 \pm 0.9$ & 0.443 \\
\hline CA19-9 (<34 U/mL) & $13.3 \pm 19.6$ & $25.7 \pm 55.1$ & 0.187 \\
\hline LDH (109-245 U/L) & $170.3 \pm 32.0$ & $225.2 \pm 186.2$ & 0.529 \\
\hline
\end{tabular}

HRLH, hepatic reactive lymphoid hyperplasia; PHL, primary hepatic lymphoma; AFP, alpha fetoprotein; CEA, carcinoembryonic antigen; CA19-9, carbohydrate antigen 19-9; LDH, lactate dehydrogenase. 


\section{Results of conventional ultrasound}

The conventional ultrasound features of HRLH and PHL were shown in Table 2. HRLH and PHL were mostly observed in the right lobe $(90.9 \%$ and $71.4 \%)$ $(P=0.604)$ and were usually under the capsule $(63.6 \%$ and $64.3 \%)(P=0.648)$. Both HRLH and PHL usually displayed the character of clear boundary $(72.7 \%$ and $64.3 \%)$, hypoechogenicity $(72.7 \%$ and $64.3 \%)$ and sometimes with the posterior echo enhancement $(45.5 \%$ and $14.3 \%)(P>0.05)$. There were also no significant differences of color Doppler flow signal and resistance index between HRLH and PHL $(P>0.05)$. But, the maximum diameter of HRLH $(16.5 \pm 3.8 \mathrm{~mm})$ was significantly smaller than that of PHL $(48.3 \pm 36.2 \mathrm{~mm})$ $(P=0.008)$. And more HRLH $(90.9 \%)$ lesions were regular in shape than PHL $(50.0 \%)(P=0.038)$ (Fig. 1).

Table 2 The conventional ultrasound manifestations of HRLH and PHL

\begin{tabular}{|c|c|c|c|}
\hline Item & $\operatorname{HRLH}(n=11)$ & $\operatorname{PHL}(n=14)$ & $P$ value \\
\hline \multicolumn{4}{|l|}{ Location $(n, \%)$} \\
\hline Right lobe & $10(90.9)$ & $10(71.4)$ & 0.604 \\
\hline Left lobe & $0(0.0)$ & $2(14.3)$ & \\
\hline Caudate lobe & $1(9.1)$ & $2(14.3)$ & \\
\hline \multicolumn{4}{|l|}{ Subcapsular lesion $(n, \%)$} \\
\hline Yes & $7(63.6)$ & $9(64.3)$ & 0.648 \\
\hline No & $4(36.4)$ & $5(35.7)$ & \\
\hline Maximum diameter (mm) & $16.5 \pm 3.8$ & $48.3 \pm 36.2$ & 0.008 \\
\hline \multicolumn{4}{|l|}{ Echotexture $(n, \%)$} \\
\hline Anechoic & $2(18.2)$ & $2(14.3)$ & 0.808 \\
\hline Hypoechoic & $8(72.7)$ & $9(64.3)$ & \\
\hline Isoechoic & $1(9.1)$ & $1(7.1)$ & \\
\hline Hyperechoic & $0(0.0)$ & $2(14.3)$ & \\
\hline \multicolumn{4}{|c|}{ Posterior echo enhancement $(n, \%)$} \\
\hline Yes & $5(45.5)$ & $2(14.3)$ & 0.102 \\
\hline No & $6(54.5)$ & $12(85.7)$ & \\
\hline \multicolumn{4}{|l|}{ Shape $(n, \%)$} \\
\hline Regular & $10(90.9)$ & $7(50.0)$ & 0.038 \\
\hline Irregular & $1(9.1)$ & $7(50.0)$ & \\
\hline \multicolumn{4}{|l|}{ Boundary $(n, \%)$} \\
\hline Clear & $8(72.7)$ & $9(64.3)$ & 0.496 \\
\hline Not clear & $3(27.3)$ & $5(35.7)$ & \\
\hline \multicolumn{4}{|c|}{ Color Doppler flow signal $(n, \%)$} \\
\hline No & $7(63.6)$ & $4(28.6)$ & 0.376 \\
\hline Dotted & $1(9.1)$ & $3(21.4)$ & \\
\hline Linear & $3(27.3)$ & $6(42.9)$ & \\
\hline Rich & $0(0.0)$ & $1(7.1)$ & \\
\hline Resistance index & $0.56 \pm 0.04$ & $0.66 \pm 0.10$ & 0.142 \\
\hline
\end{tabular}

HRLH, hepatic reactive lymphoid hyperplasia; PHL, primary hepatic lymphoma. 

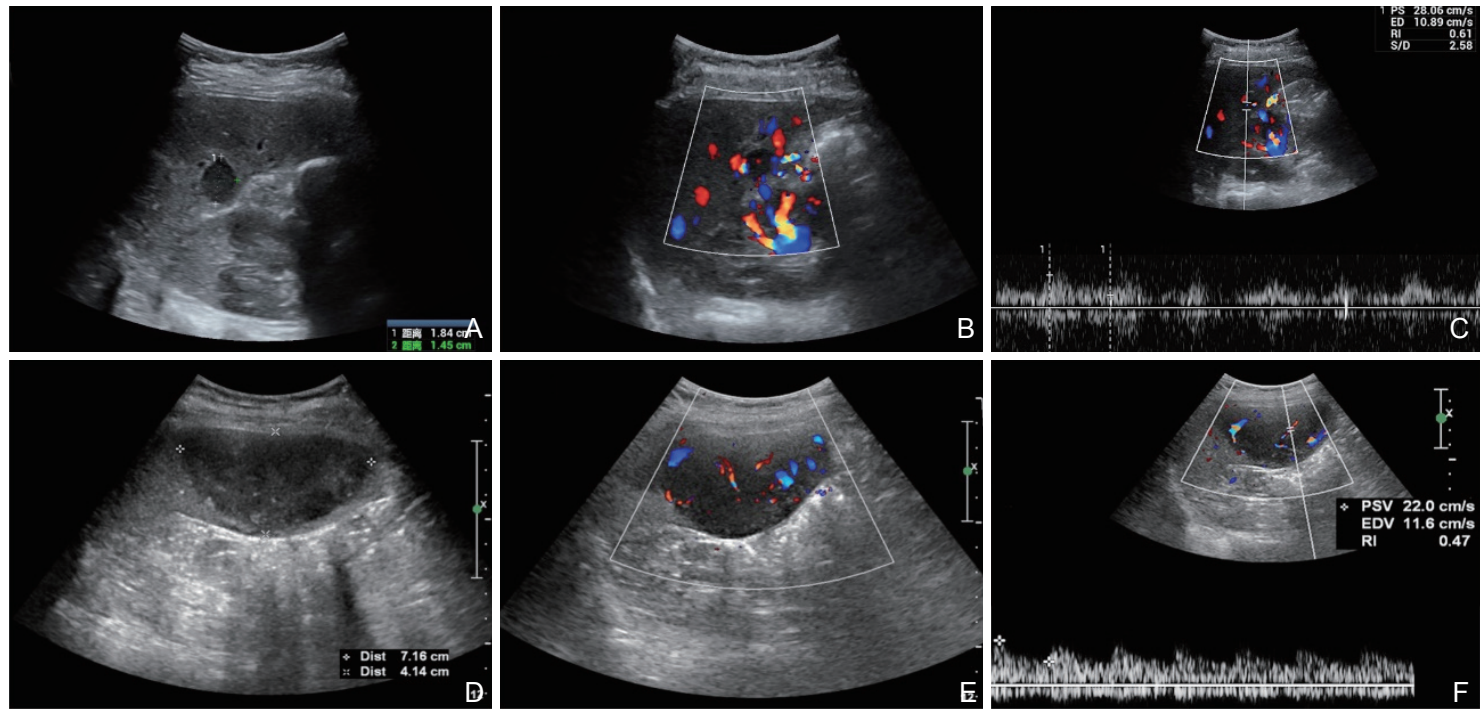

Figure 1 The conventional ultrasound images of hepatic reactive lymphoid hyperplasia (HRLH) in a 34-year-old woman (A-C) and primary hepatic lymphoma (PHL) in a 37-year-old man (D-F). (A) The subcapsular HRLH lesion in the right lobe showed hypoechogenicity with posterior echo enhancement, clear boundary and regular shape, the size of the lesion was $18 \times 15 \mathrm{~mm}$. (B) Color Doppler ultrasound showed dotted blood flow signal. (C) Resistance index was 0.61. (D) The subcapsular PHL lesion in the right lobe showed hypoechogenicity without posterior echo enhancement, clear boundary and irregular shape, the size of the lesion was $72 \times 41 \mathrm{~mm}$. (E) Color Doppler ultrasound showed rich blood flow signal. (F) Resistance index was 0.47 .

\section{Results of CEUS}

The CEUS features of HRLH and PHL were shown in Table 3. CEUS was performed on 10 patients with HRLH and 6 patients with PHL. All HRLH and PHL lesions exhibited arterial enhancement and washout in early portal phase and there were no significant differences of washin time $(12.6 \pm 3.6 \mathrm{~s}$ and $14.5 \pm 4.4 \mathrm{~s})$, time to peak $(16.9 \pm 3.1 \mathrm{~s}$ and $21.0 \pm 5.3 \mathrm{~s})$ and washout time $(26.3 \pm 7.8 \mathrm{~s}$ and $28.0 \pm 8.0 \mathrm{~s})$ between them $(P>$ $0.05)$. In the arterial phase, they all showed whole-lesion enhancement, in portal venous and late phases, they all showed hypoenhancement (Fig. 2 and Fig. 3). The
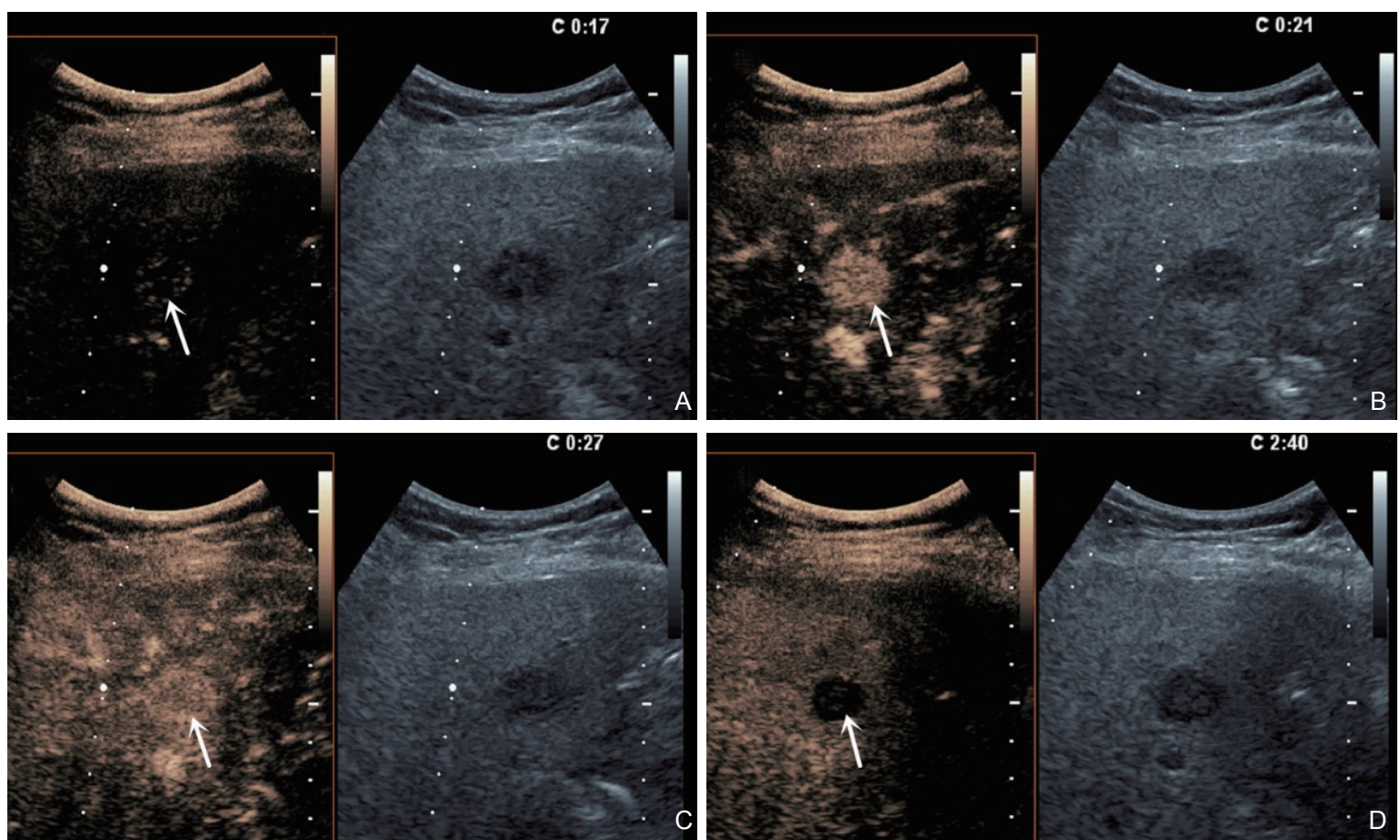

Figure 2 The contrast-enhanced ultrasound images of hepatic reactive lymphoid hyperplasia in a 57-year-old woman. (A) The lesion began to show enhancement at $17 \mathrm{~s}$ after contrast injection. (B) The peak enhancement appeared at $21 \mathrm{~s}$ after contrast injection and the lesion displayed whole hyperenhancement. (C) The contrast agent in the lesion began to wash out at $27 \mathrm{~s}$ after injection. (D) The lesion showed hypoenhancement in the late phase. The lesions were indicated by arrows. 
differences between them were that all HRLH displayed hyperenhancement (100\%) in arterial phase while PHL could be hypoenhancement (16.7\%), isoenhancement $(33.3 \%)$ and hyperenhancement $(50 \%)(P=0.036)$.
And at the beginning of washout, rim enhancement was seen more frequently in HRLH $(90 \%)$ than that in PHL $(33.3 \%)(P=0.036)$ (Fig. 4).

Table 3 The contrast-enhanced ultrasound manifestations of HRLH and PHL

\begin{tabular}{|c|c|c|c|}
\hline Item & HRLH $(n=10)$ & $\operatorname{PHL}(n=6)$ & $P$ value \\
\hline \multicolumn{4}{|c|}{ Degree of arterial phase enhancement $(n, \%)$} \\
\hline Hypoenhancement & $0(0.0)$ & $1(16.7)$ & 0.036 \\
\hline Isoenhancement & $0(0.0)$ & $2(33.3)$ & \\
\hline Washin time (s) & $12.6 \pm 3.6$ & $14.5 \pm 4.4$ & 0.364 \\
\hline Time to peak (s) & $16.9 \pm 3.1$ & $21.0 \pm 5.3$ & 0.080 \\
\hline Washout time (s) & $26.3 \pm 7.8$ & $28.0 \pm 8.0$ & 0.683 \\
\hline \multicolumn{4}{|c|}{ Rim enhancement at the beginning of washout $(n, \%)$} \\
\hline Yes & $9(90.0)$ & $2(33.3)$ & 0.036 \\
\hline No & $1(10.0)$ & $4(66.7)$ & \\
\hline
\end{tabular}

HRLH, hepatic reactive lymphoid hyperplasia; PHL, primary hepatic lymphoma.
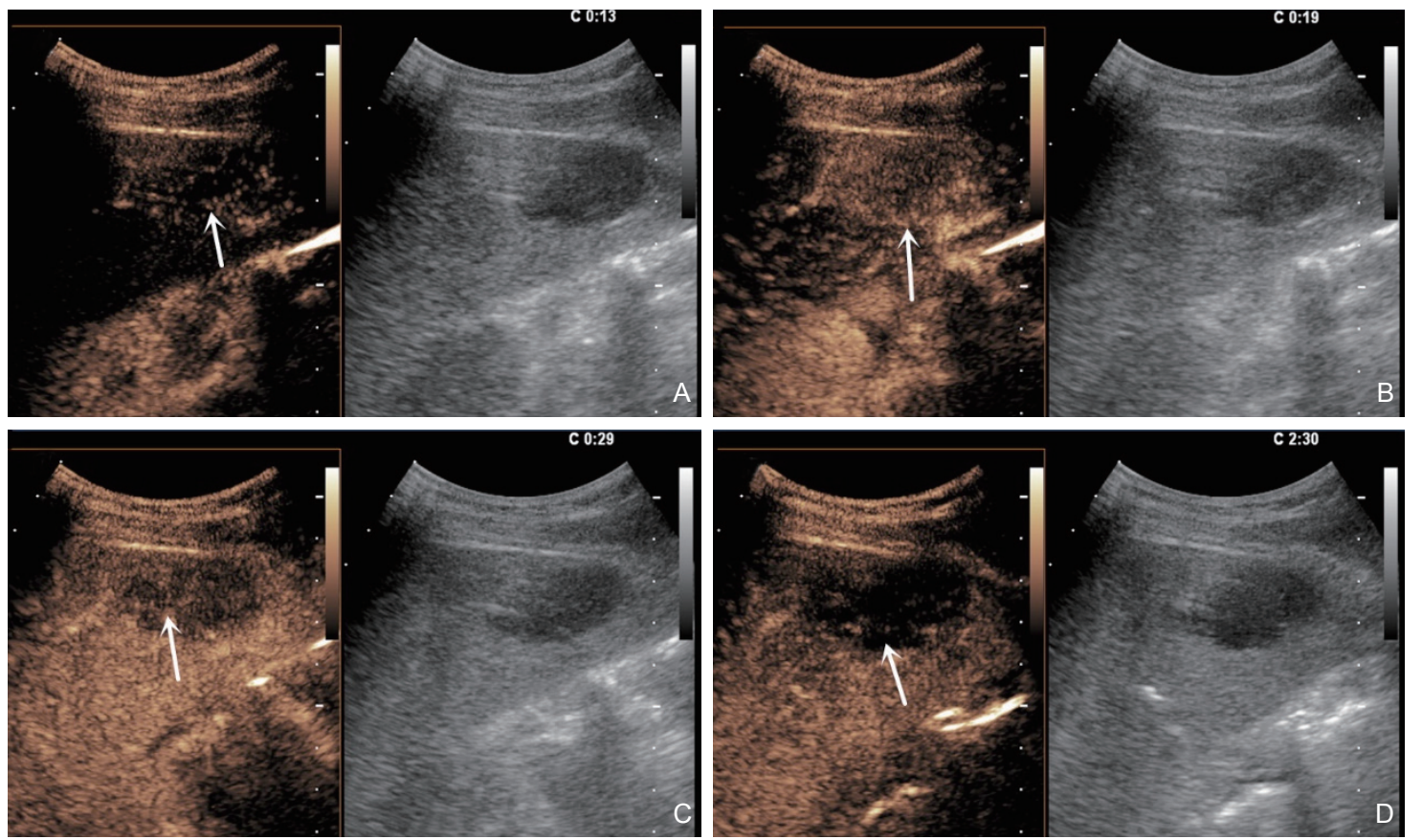

Figure 3 The contrast-enhanced ultrasound images of primary hepatic lymphoma in a 43-year-old woman. (A) The lesion began to show enhancement at $13 \mathrm{~s}$ after contrast injection. (B) The peak enhancement appeared at $19 \mathrm{~s}$ after contrast injection and the lesion displayed whole hyperenhancement. (C) The contrast agent in the lesion began to wash out at $29 \mathrm{~s}$ after injection. (D) The lesion showed hypoenhancement in the late phase. The lesions were indicated by arrows.

\section{Histopathological findings}

Microscopic evaluation of HRLH lesions revealed the formation of lymphoid follicles and germinal centers
(Fig. 5A). Immunohistochemically, T and B lymphocytes were proliferated, but the distribution was normal.

Among the 14 patients with PHL, 5 (35.7\%) patients 
were diagnosed with diffuse large B-cell lymphoma (DLBCL) and $9(64.3 \%)$ were mucosa-associated lymphoid tissue (MALT) lymphoma. There were diffuse proliferative atypical cells with mitosis in the lesion area of DLBCL (Fig. 5B). Immunohistochemistry indicated the positivity of $\mathrm{B}$ cell markers and high Ki-67 index. For MALT lymphoma, it was characterized by the infiltration of small lymphocytes with mild atypia in the interfollicular areas and lymphoepithelial changes compared with HRLH (Fig. 5C). The final diagnosis was also based on the results of EBER analysis, reticulum fiber staining, gene rearrangement and DC-FISH analysis (Table 4).
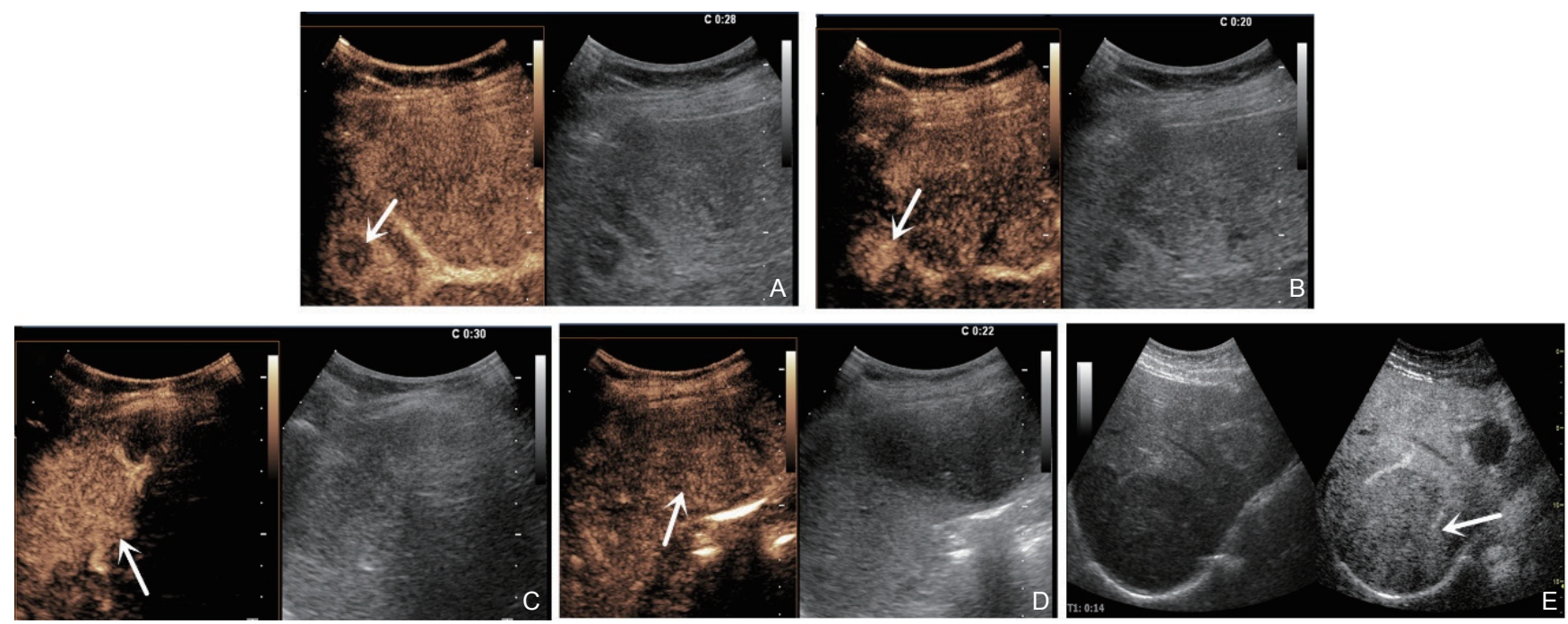

Figure 4 The comparison of contrast-enhanced ultrasound features of hepatic reactive lymphoid hyperplasia (HRLH) and primary hepatic lymphoma (PHL). (A) The characteristic rim enhancement of HRLH appeared when the contrast agent began to wash out. (B) The HRLH lesion showed hyperenhancement in arterial phase. (C) The PHL lesion showed hyperenhancement in arterial phase. (D) The PHL lesion showed isoenhancement in arterial phase; (E) The PHL lesion showed hypoenhancement in arterial phase. The lesions were indicated by arrows.
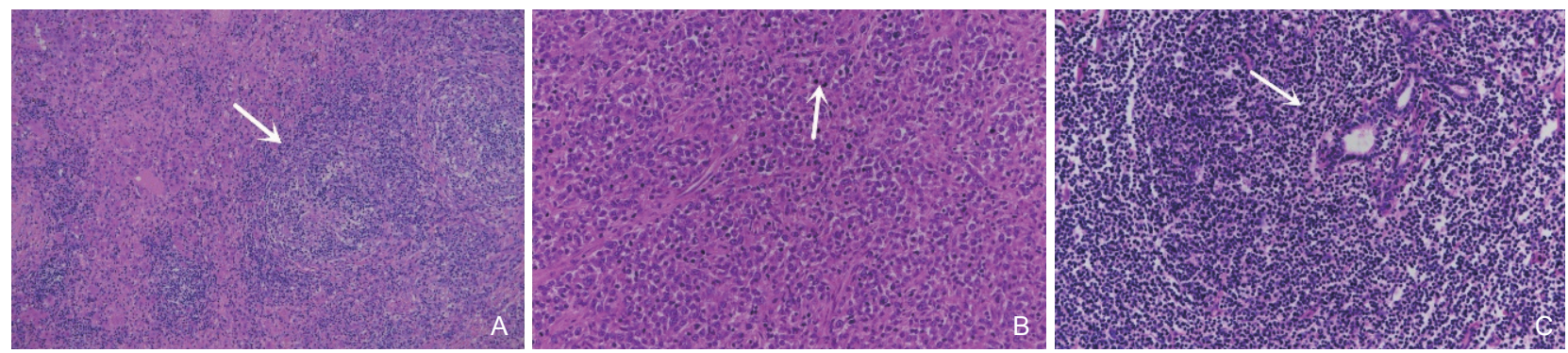

Figure 5 Histological images of hepatic reactive lymphoid hyperplasia (A), diffuse large B-cell lymphoma (B) and mucosa-associated lymphoid tissue lymphoma $(\mathrm{C})$ (hematoxylin-eosin stain, $\times 100$ ). A, The nodule was consisted of proliferated lymphocytes with the formation of lymphoid follicles and germinal centers (arrow). B, Diffuse infiltration of atypical large lymphocytes (arrow). C, Mildly atypical lymphocytes and lymphoepithelial change (arrow) could be seen in the nodule.

\section{Discussion}

Reactive lymphoid hyperplasia is a kind of nonneoplastic lesion that can be found in the lung, gastrointestinal tract, pancreas and breast, but it is peculiar in the liver and most of the studies about HRLH are case reports [13]. PHL is an uncommon malignant neoplasm of the liver, representing less than $1 \%$ of extranodal lymphomas [14]. Although HRLH and PHL are all lymphoproliferative disorders, the therapy and prognosis are dramatically different, it is quite necessary to make an accurate differentiation diagnosis of them. However, on account of the rarity and pathological similarity of them, the preoperative identification is a great challenge.

Ultrasound is the first choice for screening of focal liver lesions, which gives the information of liver parenchyma, nodule location, size and blood flow information with conventional ultrasound. However, further examination is usually needed to achieve a correct diagnosis. CEUS can image the microcirculation of the nodule and adjacent liver tissue in real time thus 
bears remarkable advantages in discriminating benign and malignant tumors $[15,16]$. But to our knowledge, no multi-sample research specially analyzing the ultrasound differentiation diagnosis of HRLH and PHL has been reported, and our study is the first one concentrating on this point.

Table 4 The results of EBER analysis, reticulum fiber staining, gene rearrangement and DC-FISH analysis

\begin{tabular}{|c|c|c|c|}
\hline Item & HRLH & DLBCL & MALT lymphoma \\
\hline \multicolumn{4}{|l|}{$\operatorname{EBER}(n, \%)$} \\
\hline Positive & $0(0.0)$ & $1(33.3)$ & $1(20.0)$ \\
\hline Negative & $6(100.0)$ & $2(66.7)$ & $4(80.0)$ \\
\hline \multicolumn{4}{|c|}{ Reticulum fiber staining $(n, \%)$} \\
\hline Obvious hyperplasia & $0(0.0)$ & $1(100.0)$ & $3(75.0)$ \\
\hline No hyperplasia & $2(100.0)$ & $0(0.0)$ & $1(25.0)$ \\
\hline \multicolumn{4}{|c|}{ Gene rearrangement $(n, \%)$} \\
\hline Positive & $0(0.0)$ & $1(50.0)$ & $4(100.0)$ \\
\hline Negative & $4(100.0)$ & $1(50.0)$ & $0(0.0)$ \\
\hline \multicolumn{4}{|l|}{ DC-FISH $(n, \%)$} \\
\hline Positive & $0(0.0)$ & $0(0.0)$ & $1(20.0)$ \\
\hline Negative & $3(100.0)$ & $1(100.0)$ & $4(80.0)$ \\
\hline
\end{tabular}

EBER, Epstein-Barr virus-encoded small RNA; DC-FISH, dual-color fluorescence in situ hybridization; HRLH, hepatic reactive lymphoid hyperplasia; DLBCL, diffuse large B-cell lymphoma; MALT, mucosa-associated lymphoid tissue.

In this study, HRLH and PHL lesions display many nonspecific features in the conventional ultrasound. Firstly, both of them are usually observed in the right lobe, and about $60 \%$ of the lesions are under the capsule, this may attribute to the lesion's growing along the terminals of blood vessels $[13,17,18]$. Secondly, the two kinds of lesions are generally hypoechoic and sometimes anechoic accompanied with posterior echo enhancement in the gray scale ultrasound images, the reason is that the nodules are composed of homogeneous lymphocytes which leads to small acoustic impedance difference and reduced acoustic attenuation [19-22]. It is worth noting that all the HRLH lesions in the study are $\leq 2 \mathrm{~cm}$ in size which is compatible with other reports [8, 23-25], while PHL is larger, and the irregular shape may be related with its large size.

In the CEUS images, HRLH and PHL show wholelesion enhancement in arterial phase, the contrast agent begins to wash in at the early stage of arterial phase, then reaches the peak rapidly and begins to wash out before $30 \mathrm{~s}$ after injection of a contrast agent. In all, HRLH and PHL present "quick washin and quick washout" enhancement pattern. This is probably because that the nodule is mainly composed of lymphocytes and less interstitial tissues, the blood drainage is unobstructed, so the contrast agent can quickly wash in and then quickly wash out with the blood flow.

However, there are pivotal clues to facilitate differentiation in CEUS findings. In the first place, HRLH is hyperenhanced in arterial phase while PHL can be hypoenhanced, isoenhanced and hyperenhanced, the difference may be correlated with the different microvessel density inside the lesions. Another significant difference is that $90 \%$ of HRLH lesions show rim enhancement at the beginning of washout when only $33.3 \%$ of PHL lesions have this sign. Many studies have also reported perinodular enhancement of HRLH on contrast-enhanced CT and MRI [9,26-28]. There are some pathological hypotheses about this manifestation. Yoshida et al presume that the lymphocytic infiltration in the portal tracts around the nodule causes portal flow disturbance and increased hepatic arterial flow in the perinodular liver parenchyma, thus leading to perinodular enhancement [28]. Sonomura et al consider that the sinusoidal dilatation surrounding the nodule may be the cause of the perinodular enhancement [9] Dong et al hold the hypothesis of arterial-portal venous shunt, thinking that on the one hand, the infiltration of lymphocytes may result in the compression of portal branches followed by the compensated increase of 
arterial blood flow. On the other hand, in the lesion, the sinusoids and central veins may be stenotic and the normal blood drainage is blocked, then the portal branches become the drainage vessels instead of supplying vessels, thus the HRLH lesion washes out quickly, while the drainage route remains normal in the perinodular parenchyma. In consequence, the increased peripheral arterial blood flow, the quick wash out of the HRLH lesion and the relatively normal wash out of the perinodular parenchyma make the rim enhancement an important hallmark of HRLH [29]. PHL can also occasionally present this phenomenon, Maher et al attribute it to vasculitis induced in the adjacent liver parenchyma [30]. Of course, these hypotheses need to be confirmed by further pathological evidences.

The ultrasound findings of HRLH and PHL often suggest malignant neoplasms, it is necessary to distinguish them from other liver malignancies. (i) Hepatocellular carcinoma (HCC) has the highest incidence in hepatic malignant tumors, most cases reported previously are misdiagnosed as HCC. Although "quick washin and quick washout" is as well the classical feature of HCC, the washout time of HCC is usually later than 60 s compared with surrounding cirrhotic liver, which is different from that of HRLH and PHL. In addition, many patients have the hepatitis background and high AFP level. (ii) Like HRLH and PHL, the metastasis is usually detected under the capsule, but sometimes the nodule presents "bull eye" sign. During CEUS imaging, the rim enhancement often occurs in arterial phase rather than washout period because of central necrosis, and the rim is thicker than that of HRLH. The malignant tumor history and elevated serum CA19-9 or CEA can also help to make the diagnosis. (iii) The irregular rim arterial phase hyperenhancement appears in intrahepatic cholangiocarcinoma as well, which is based on the histological characteristic that the cancer cells are accumulated in the periphery of the nodule while the interior is fibrous connective tissue. The cholangiectasis and stones inside or around the lesion, plus elevated serum CA19-9 or CEA can make the diagnosis easier.

There are some limitations in the study. First, we had a small sample size of HRLH and PHL because of their rarity, more cases are needed to confirm the results and the relationship between ultrasound findings and pathological characteristics. Second, we did not analyze the CT and MRI images of HRLH and PHL, the diagnostic capability of ultrasound, CT and MRI could be compared so that the clinician can choose the most appropriate modality.

In conclusion, although HRLH and PHL are rare diagnoses, they should be considered when a nodule displays marked hypoechogenicity or accompanied with posterior echo enhancement in conventional ultrasound, and presents whole-lesion arterial phase enhancement, quick washin and especially quick washout in CEUS. If the lesion is $\leqslant 2 \mathrm{~cm}$ in size, displays regular shape and rim enhancement at the beginning of washout, it is more likely to be HRLH. Otherwise, if the lesion is larger, presents irregular shape and no obvious rim enhancement at the beginning of washout, it is probably PHL. Ultrasound can provide valuable diagnostic clues in the diagnosis and differentiation of HRLH and PHL.

\section{Acknowledgments}

This work was supported by National Natural Science Foundation of China (No. 81873897).

\section{Conflicts of Interest}

The authors declare no conflicts of interest.

\section{References}

[1] Song KD, Jeong WK. Benign nodules mimicking hepatocellular carcinoma on gadoxetic acid-enhanced liver MRI. Clin Mol Hepatol 2015; 21: 187-191.

[2] Zhang W, Zheng S. Reactive lymphoid hyperplasia of the liver: A case report. Medicine (Baltimore) 2019; 98: e16491.

[3] Calvo J, Carbonell N, Scatton O, Marzac C, Ganne-Carrie N, Wendum D. Hepatic nodular lymphoid lesion with increased IgG4positive plasma cells associated with primary biliary cirrhosis: a report of two cases. Virchows Arch 2015; 467: 613-617.

[4] Ippolito D, Porta M, Maino C, Pecorelli A, Ragusi M, Giandola T, et al. Diagnostic approach in hepatic lymphoma: radiological imaging findings and literature review. J Cancer Res Clin Oncol 2020; 146:1545-1558.

[5] Zhang SL, Chen C, Rao QW, Guo Z, Wang X, Wang ZM, et al. Incidence, prognostic factors and survival outcome in patients with primary hepatic lymphoma. Front Oncol 2020; 10: 750.

[6] Rajesh S, Bansal K, Sureka B, Patidar Y, Bihari C, Arora A. The imaging conundrum of hepatic lymphoma revisited. Insights Imaging 2015; 6:679-692.

[7] Padhan RK, Das P, Shalimar. Primary hepatic lymphoma. Trop Gastroenterol 2015; 36: 14-20.

[8] Zhou Y, Wang X, Xu C, Zhou GF, Zeng MS, Xu PJ. Hepatic pseudolymphoma: imaging features on dynamic contrast-enhanced MRI and diffusion-weighted imaging. Abdom Radiol (NY) 2018; 43:2288-2294.

[9] Sonomura T, Anami S, Takeuchi T, Nakai M, Sahara S, Tanihata $\mathrm{H}$, et al. Reactive lymphoid hyperplasia of the liver: Perinodular enhancement on contrast-enhanced computed tomography and magnetic resonance imaging. World J Gastroenterol 2015; 21:67596763.

[10] Bai Y, Liang W. Potential value of apparent diffusion coefficient in the evaluation of hepatic pseudolymphoma. Quant Imaging Med Surg 2019; 9:340-345.

[11] Colagrande S, Calistri L, Grazzini G, Nardi C, Busoni S, Morana G, et al. MRI features of primary hepatic lymphoma. Abdom Radiol (NY) 2018; 43:2277-2287.

[12] CEUS LI-RADS® v2017 CORE. ACR, 2017. 
[13] Kwon YK, Jha RC, Etesami K, Fishbein TM, Ozdemirli M, Desai CS. Pseudolymphoma (reactive lymphoid hyperplasia) of the liver: A clinical challenge. World J Hepatol 2015; 7:2696-2702.

[14] Mezzano G, Rojas R, Morales C, Gazitúa R, Díaz JC, Brahm J. Primary hepatic lymphoma: An infrequent focal liver tumour. Gastroenterol Hepatol 2016; 39:674-676.

[15] Durot I, Wilson SR,Willmann JK. Contrast-enhanced ultrasound of malignant liver lesions. Abdom Radiol (NY) 2018; 43:819-847.

[16] Schellhaas B, Strobel D. Tips and tricks in contrast-enhanced ultrasound (CEUS) for the characterization and detection of liver malignancies. Ultraschall Med 2019; 40:404-424. English.

[17] Seitter S, Goodman ZD, Friedman TM, Shaver TR, Younan G. Intrahepatic reactive lymphoid hyperplasia: A case report and review of the literature. Case Rep Surg 2018; 2018: 9264251.

[18] Yang CT, Liu KL, Lin MC, Yuan RH. Pseudolymphoma of the liver: Report of a case and review of the literature. Asian J Surg 2017; 40:74-80.

[19] Gu LS, Cui NY, Wang Y, Che SN, Zou SM, He W, et al. Comparison of sonographic characteristics of primary thyroid lymphoma and anaplastic thyroid carcinoma. J Thorac Dis 2017; 9:4774-4784.

[20] Asai S, Miyachi H, Kawakami C, Kubota M, Kato Y, Shimamura $\mathrm{K}$, et al. Infiltration of cervical lymph nodes by B- and T-cell nonHodgkin's lymphoma and Hodgkin's lymphoma: preliminary ultrasonic findings. Am J Hematol 2001; 67:234-239.

[21] Gupta A, Rahman K, Shahid M, Kumar A, Qaseem SM, Hassan SA, et al. Sonographic assessment of cervical lymphadenopathy: role of highresolution and color Doppler imaging. Head Neck 2011; 33:297-302.

[22] Asai S, Okami K, Nakamura N, Ogawa Y, Ohta Y, Ogase Y, et al. The tortoiseshell pattern in one or both sides of the submandibular glands in mucosa-associated lymphoid tissue lymphoma is related to chromosomal aberrations and the disease extent. $J$ Ultrasound Med 2010; 29:111-115.

[23] Inoue M, Tanemura M, Yuba T, Miyamoto T, Yamaguchi M, Irei T, et al. A case of hepatic pseudolymphoma in a patient with primary biliary cirrhosis. Clin Case Rep 2019; 7:1863-1869

[24] Kunimoto H, Morihara D, Nakane SI, Tanaka T, Yokoyama K, Anan A, et al. Hepatic pseudolymphoma with an occult hepatitis B virus infection. Intern Med 2018; 57:223-230.

[25] Takahashi Y, Seki H, Sekino Y. Pseudolymphoma with atrophic parenchyma of the liver: Report of a case. Int J Surg Case Rep 2018; 49: 136-139.

[26] Fukuo Y, Shibuya T, Fukumura Y, Mizui T, Sai JK, Nagahara A, et al. Reactive lymphoid hyperplasia of the liver associated with primary biliary cirrhosis. Med Sci Monit 2010 ;16:CS81-86.

[27] Tuckett J, Hudson M, White S, Scott J. Reactive lymphoid hyperplasia of the liver: A case report and review of imaging characteristics. Eur J Radiol Extra 2011; 79: e11-14.

[28] Yoshida K, Kobayashi S, Matsui O, Gabata T, Sanada J, Koda W, et al. Hepatic pseudolymphoma: imaging-pathologic correlation with special reference to hemodynamic analysis. Abdom Imaging 2013; 38:1277-1285.

[29] Dong CH, Lu Q, Wang WP, Ji ZB, Wang X. Contrast-enhanced ultrasound features of hepatic reactive lymphoid hyperplasia: correlation with histopathologic findings. J Ultrasound Med 2019; 38:2379-2388.

[30] Maher MM, Mcdermott SR, Fenlon HM, Conroy D, O'Keane JC, Carney DN, et al. Imaging of primary non-Hodgkin's lymphoma of the liver. Clin Radiol 2001; 56:295-301. 